\title{
STUDY OF ENZYMATIC ANTIOXIDANT MARKER \& LIPID PEROXIDATION STATUS IN PATIENTS WITH OSTEOARTHRITIS
}

Manoj Narayan Paliwal, A. N. Sontakke, Prachi Paliwal

1. Assistant Professor, MD, Department of Biochemistry, R. D. Gardi Medical College, Ujjain.

2. Professor \& Head, MD, Department of Biochemistry, MIMER Medical College, Pune.

3. Demonstrator, Department of Biochemistry, R. D. Gardi Medical College, Ujjain.

CORRESPONDING AUTHOR

Dr. Manoj Narayan Paliwal (Biochemistry Dept.)

R. D. Gardi Medical College, Agar Road,

Surasa, Ujjain (M.P.) Pin- 456003,

E-mail: dr.manojpaliwal@yahoo.co.in,

Ph: $009109303158744,00919977692204$.

ABSTRACT: BACKGROUND/AIMS: Osteoarthritis is an inflammatory \& degenerative disorder of joints. The exact pro-oxidant $\&$ antioxidant status is not clear in osteoarthritis. Our aims were to estimate levels of the lipid peroxidation (in terms of MDA) \& enzymatic antioxidant (in terms of superoxide dismutase) in serum of osteoarthritis patients \& compare them with the levels in normal healthy controls. MATERIAL \& METHODS: A study was performed at the department of biochemistry at Pd. Dr. D. Y. Patil medical college, Pimpri, Pune-18(M.S.) In 30 patients of osteoarthritis serum levels of enzymatic antioxidant Marker (SOD) \& lipid peroxidation status (MDA) were estimated by spectrophotometry. Thirty healthy controls were also included in the study \& serum levels of same parameters were also measured. STATISTICAL ANALYSIS: It was performed by using the student unpaired $t$ test. RESULT: A serum level of enzymatic antioxidant marker (SOD) was increased in the patients than in the controls. The serum lipid peroxidation (MDA) level was increased in the patients than in the controls.

KEYWORDS: Osteoarthritis (OA), Superoxide dismutase (SOD), Malondialdehyde (MDA)

INTRODUCTION: Osteoarthritis is a degenerative \& inflammatory, joint disease, characterized by progressive erosion of articular cartilage. Osteoarthritis joins heart disease \& cancer as one of the dividens of growing older.The age related changes in cartilage include alterations in proteoglycans \& collagen .Chondrocyte plays an important role in the process \& plays a cellular basis of the disease. ${ }^{1}$

At the sites of inflammation of joint increased free radical activity is associated with activation of neutrophils, phagocytosis by macrophages, which involve respiratory bursts phenomenon\& uncoupling of variety of cellular redox systems. ${ }^{2,3} \&$ lead to ultimately increased peroxidation of unsaturated lipids of the membrane.

Lipid peroxidation mediated by free radicals is considered to be the major mechanism of cell membrane destruction \& cell damage. Antioxidants are compounds that dispose, scavenge, $\&$ suppress the formation of free radicals or oppose their actions. ${ }^{4}$

This study evaluates the association between lipid Peroxidation \& enzymatic antioxidant marker in osteoarthritis.

To the best of our knowledge, only very few studies have been performed with respect to the estimation of the serum enzymatic antioxidant levels in osteoarthritis \& their role in prevention \& treatment of osteoarthritis. In the light of this explanation, the present study was 
undertaken to find out the levels of the enzymatic antioxidant (SOD) in the serum of osteoarthritis.

MATERIAL \& METHOD: This study was conducted in the department of biochemistry, Pd. Dr. D. Y. Patil medical college, Pimpri. Pune-18.

The present study consists of thirty clinically diagnosed cases of osteoarthritis ranging in the age from 30 to 60 years. The control group consists of age \& sex matched thirty healthy volunteer.

A thorough physical examination was carried out on all the patients. Routine hematological \& radiological investigation was also done. Thirty cases selected from orthopedics OPD diagnosed by orthopedician. The presence of osteoarthritis in patients were diagnosed by carrying out x-ray analysis of joint destruction as well as $\mathrm{C}$-reactive protein \& antinuclear antibodies test.

INCLUSION CRITERIA: subjects with normal nutritional habits without supplementing with any vitamins during the last six months included in the study.

EXCLUSION CRITERIA - None of these subjects were alcoholics or chronic smokers, \& none of them suffered from any systemic diseases like hypertension, diabetes, (confirmed by clinical \& laboratory examination), not having any history of trauma to the joints, also subjects with history of receiving anti-inflammatory drugs in the last six months were excluded from study. This study was also approved by the institutional ethical committee.

$3 \mathrm{ml}$ of fasting venous blood samples were collected in Plain vials for estimation of MDA. $\&$ SOD.

Serum for MDA \&SOD estimation was separated by centrifuging the blood at $3000 \mathrm{rpm}$ for 10 minute.

Serum MDA was estimated by Thiobarbituric acid method.$^{5}$

SOD (Superoxide dismutase) was estimated by Markland \& Markland method . 6

All estimations were done within 24-48 hrs after specimen collection.

The results were presented as Mean \pm SD. Statistical analysis was performed by using the student unpaired t- test $\&$. P value $<0.05$ was considered as significant.

RESULT: In present study, serum Malondialdehyde, Superoxide dismutase levels were estimated

\section{$P<0.05$ compared to controls - considered as a statistically significant}

The serum MDA levels in the osteoarthritis patient was $9.37 \pm 5.33 \mathrm{nmol} / \mathrm{ml}$ which was increased than that of controls $(3.98 \pm 1.98 \mathrm{nmol} / \mathrm{ml}, \mathrm{P}<0.05)$

Serum SOD levels in the osteoarthritis patient was $4.94 \pm 3.0 \mathrm{unit} / \mathrm{ml}$ which was increased in OA patients than controls $(3.31 \pm 0.85, \mathrm{P}<0.05)$

DISCUSSION: Osteoarthritis is characterized by increased markers of oxidative stress.

Recent studies have suggested that human articular chondrocyte can actively produce reactive oxygen species. (ROS). ${ }^{7}$ 
At the site of inflammation increased free radical activity is associated with activation of neutrophils, phagocytosis by macrophages which involve respiratory "burst phenomenon" and uncoupling of variety of cellular redox systems. These processes lead to ultimately increased peroxidation of unsaturated lipids of membrane. Increased lipid peroxidation suggests presence of increased oxidative stress which is the causative factor of joint diseases (K. K. Mane et al 1999). ${ }^{8}$

ROS are released during inflammation of the Synovial membrane of synoviocytes. These radical oxygen species with oxidative activity play an important role in the chondrocyte catabolic program being the mediators and effecters of cartilage damage. The damaging effect of the process is initiated by a chain reaction that provides continue supply of free radicals which initiates further peroxidation. This involves the mechanism of oxidative decomposition of $n-3$ and n-6 PUFA membrane phospholipids leading to formation of complex mixtures of lipid hydroperoxide aldehydic end products such as MDA. ${ }^{9}$

The present study showed increased level of lipid peroxidation product MDA \& increased level of enzymatic antioxidant marker superoxide dismutase (SOD).

Since MDA is an index of lipid peroxidation, its level was estimated in patients with osteoarthritis to estimate the extent of lipid peroxidation. MDA levels were found to be increased in osteoarthritis than in healthy individuals, indicating an increase in the process of lipid peroxidation in osteoarthritis. Results are in agreement with Ruby K B. I et al (1998), ${ }^{10}$ and K. K. Mane et al (1999) ${ }^{08}$ and Tiku et al (2000 \& 2003). 11,12

Serum SOD levels were found to be increased in patients with osteoarthritis than in healthy subjects indicating that increased enzymatic antioxidant levels in OA may be an adaptive response to increased oxidative stress. The over expression of SOD might be an adaptive response, and it results in increased dismutation of superoxide anion against hydrogen peroxide. Results are in agreement with M Manesh et al (2005), ${ }^{13}$

\& suprapaneni Krishna Mohan (2007). ${ }^{14}$

From the above discussion it is presumed that oxidative stress involved in pathogenesis of $\mathrm{OA}$ which results due to increased free radical production. This leads to alteration in the antioxidant status which varies with the individual antioxidant depending upon their biochemical action.

Further research required in this area to know the status of other antioxidant marker (like Vit E, C, B carotene, reduced glutathione) \& about their beneficial therapeutic effect in the management of osteoarthritis \& its complications.

\section{REFERENCES:}

1. Cotran kumar, Collinis. Bones, joints and soft tissue tumors. Robbins pathologic basis of disease $6^{\text {th }}$ edition. , page No.1246-1248.

2. Shin H.C., Hwang H J,Kang K J, Lee B H. An antioxidative and anti-inflammatory agent for potential treatment of osteoarthritis. Ecklonia cava. Arch Pharm Resp. 2006 Feb. [2]:165-71.

3. Sidhaye A, Cheskin L J. Pharmacological treatment of obesity. Adv. Psychosom med. 2006, 27:42-52.

4. Sie H. (1991).Oxidative stress: from basic research to clinical application. Am.J.Med 9, 31-38.

5. Wilbur KM, Bernheim F, ShapiroOW. The thiobarbituric acid method for Malondialdehyde estimation. Arch Biochem Biophys 1943; 250 pg No. 305 - 13. 
6. Marklund S, Markuland G. A simple assay for superoxide dismutase using autooxidation of pyrogallol. European Journal Biochem. 1974: page No, 469-72.

7. Loeser R F, Shakoor N. Aging or osteoarthritis which is the problem. Rheum Dis. Clin North Am; 29[4]: 653-673.

8. Mane K. K., Sardeshmukh A. S, Rathi D. B, Suryakar A. N. Lipid peroxide and Antioxidants in arthritis. Medical Journal of western India (1999); vol. 27: 108 110.

9. Brunella G, Lina R, Mauro F. Enhanced lipid peroxidation in synoviocytes from patient with OA. J. Rheumatol. 2003 Feb; 30[2]: 345-347.

10. Rubyk BI, Fil chagin NM, Sabadyshin RA. Change in lipid peroxidation in patient with primary osteoarthosis deformans. Ter Arkh 1988; 60 (9): $110-113$.

11. Moti L, Tiku, Shah Rahul, Allison G. T. Evidence linking chondrocyte lipid peroxidation to cartilage matrix protein degradation. The Journal of Biological chemistry 2000; 275 (26): 20069 - 20076.

12. Tiku M L, Allison G T, Naik K, Karry S K . Malondialdehyde oxidation of cartilage by chondrocytes. Osteoarthritis cartilage 2003 Mar; 11[3]: 159-166.

13. Maneesh M, Jayalekshmi H, Suma T, Chatterjees, Singh T A. Evidence for oxidative stress in osteoarthritis. Indian journal of clinical Biochemistry; 2005; 20[1]: 129-130.

14. Suprapaneni Krishna Mohan, Venkataraman G . Status of lipid peroxidation, glutathione, ascorbic acid, vitamin $\mathrm{E}$ and antioxidant enzymes in patients with osteoarthritis. Indian journal of medical sciences 2007; 61 [1]: 9-14.

Table 1: Levels of serum MDA, SOD in patients with osteoarthritis and controls.

\begin{tabular}{|l|c|c|c|}
\hline \multicolumn{1}{|c|}{ Study group } & Sample size $(\mathbf{n})$ & $\begin{array}{c}\text { MDA (nmol/ml) Mean } \\
\pm \text { SD }\end{array}$ & Unit/ml. \\
& & & Mean \pm SD \\
\hline Patients & 30 & $9.37 \pm 5.33$ & $4.94 \pm 3.00$ \\
\hline Controls & 30 & $3.98 \pm 1.98$ & $3.31 \pm 0.85$ \\
\hline
\end{tabular}


Graph-1 Serum MDA level in Osteoarthritis Patients \& Controls (MDA conc. in nmol/ml)

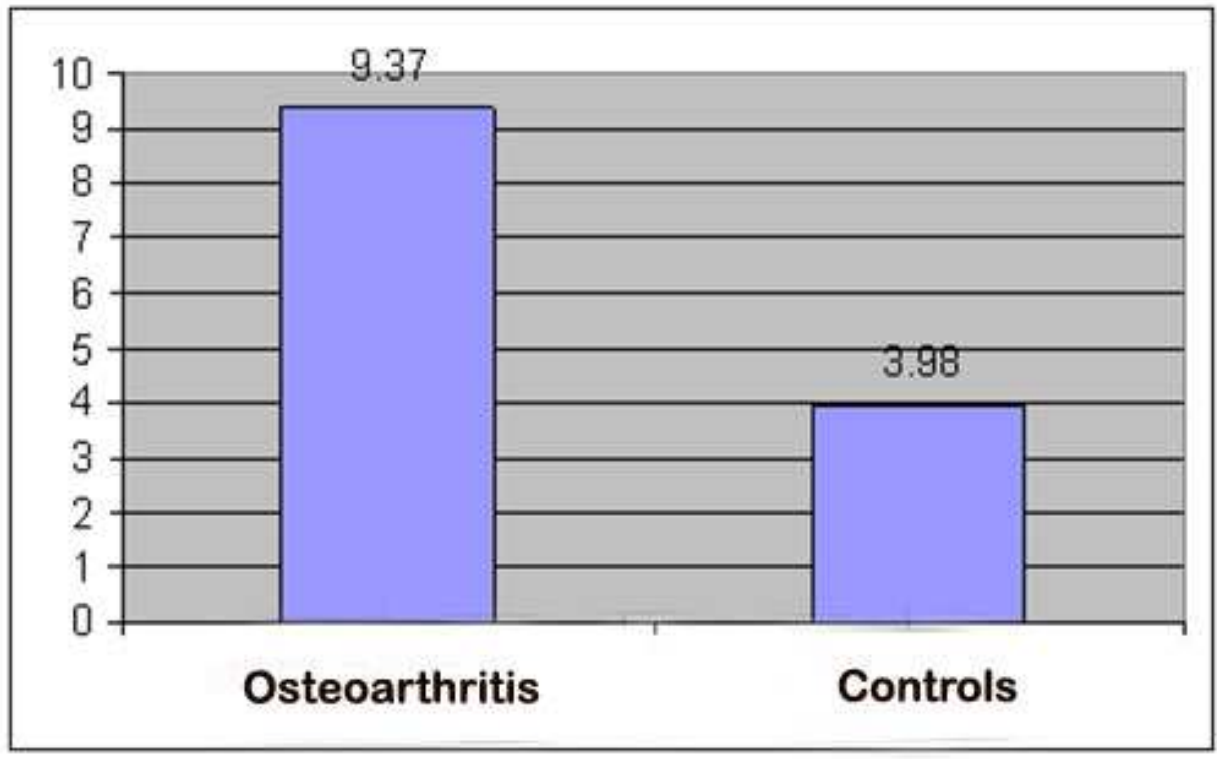

Graph-2 Serum SOD levels in osteoarthritis patients and controls.

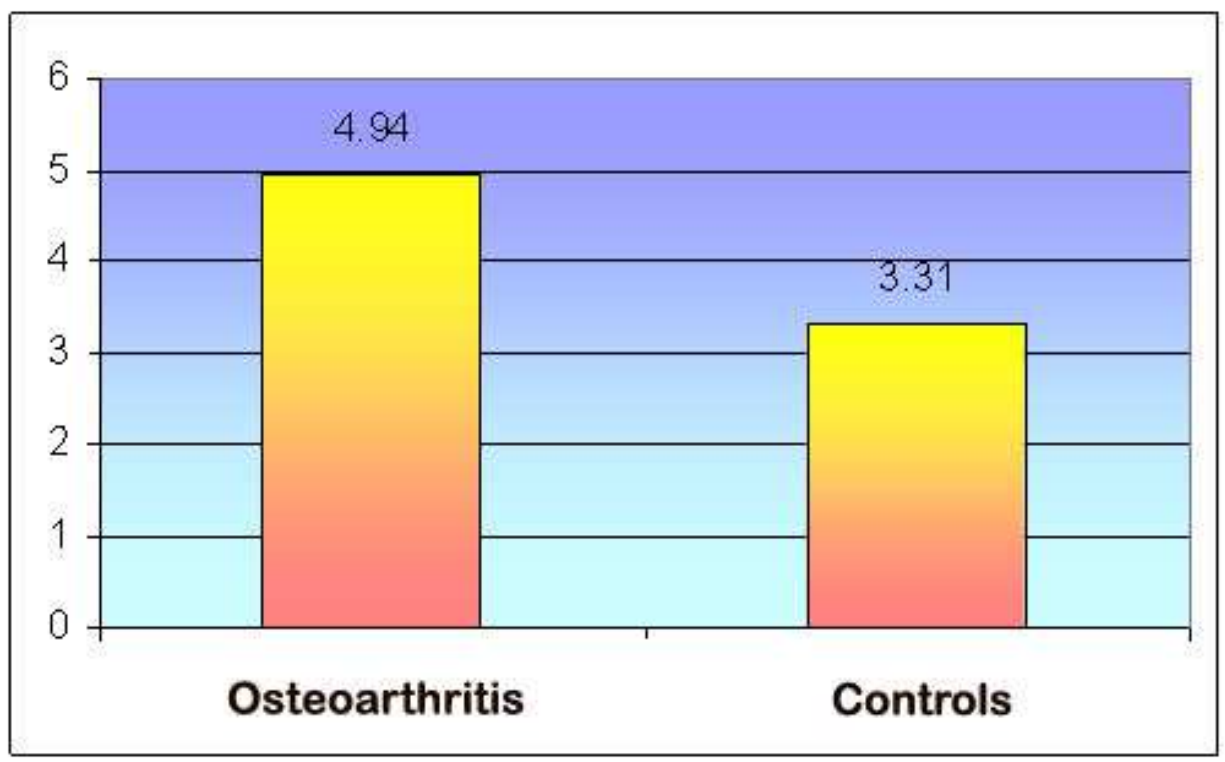

SOD Concentration unit/ml 